\title{
HABILIDADES PREDICTORAS DE ÉXITO EN EL APRENDIZAJE INICIAL DE LA LECTURA Y SU RELACIÓN CON DOS MÉTODOS DE ENSEÑANZA
}

\author{
Ma Isabel Marí-Sanmillán \\ Departamento de Ciencias de la Educación \\ Universidad CEU Cardenal Herrera \\ Castellón de la Plana \\ maria.mari1@uchceu.es \\ Ma Dolores Gil Llario \\ Departamento de Psicología Evolutiva y de la Educación \\ Universidad de Valencia \\ Estudio General. \\ dolores.gil@uv.es \\ Roberta Ceccato \\ Profesora colaboradora en la Universidad \\ Internacional de Valencia (VIU) \\ roberta.ceccato@campusviu.es
}

Fecha de Recepción: 7 Abril 2019

Fecha de Admisión: 30 Abril 2019

\section{RESUMEN}

El estudio de los factores que influyen en la adquisición de la lectura facilita la detección temprana de las dificultades del aprendizaje lector. Destacan como habilidades predictoras: el conocimiento fonológico, el conocimiento alfabético y la velocidad de denominación. Todas ellas se adquieren a lo largo de la escolaridad pero no tienen el mismo grado de implicación en las distintas fases del aprendizaje lector. Esto parece depender, en parte, del método de enseñanza de la lectura utilizado. Los objetivos de este estudio han sido identificar y analizar las habilidades implicadas en el inicio de la lectoescritura en Educación Infantil que predicen el nivel de comprensión lectora en Educación Primaria, y conocer el peso diferencial que la conciencia fonológica, la velocidad de nombramiento y otras habilidades implicadas en el proceso lector tienen durante los primeros años de instrucción en función del método empleado. Para ello se realizó un estudio longitudinal en el que participaron 130 alumnos de colegios de Valencia y Castellón. El 52\% fue iniciado con un método fonológico-sintético y el $48 \%$ con un método global. Se tomaron medidas en $2^{\circ}$ de Educación

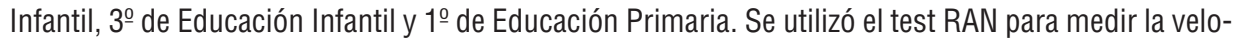
cidad de nombramiento, la Batería BIL de Inicio a la Lectura y el Test de Comprensión Lectora ACL. 
El modelo de ecuación estructural ha mostrado que en ambos métodos la velocidad de nombramiento a los 4 años tiene un efecto significativo en las variables relacionadas con el inicio de la lectura, evaluadas a los 5 años, las cuales a su vez influirían en la comprensión lectora de dichos niños a los 6 años. Esto indica que la velocidad de nombramiento constituye el cimiento del proceso de codificación fonológica sobre el que se asienta el proceso lector.

Palabras clave: conciencia fonológica; conocimiento alfabético; velocidad de nombramiento; aprendizaje de la lectura; RAN

\begin{abstract}
Predictive skills of success in the initial learning of reading and its relationship with teaching methods. The study of the factors that influence the acquisition of reading facilitates the early detection of reading learning difficulties. Phonological awareness, alphabetic knowledge, naming speed has been highlighted as predictive skills. All of them are acquired throughout school but they do not have the same degree of involvement in the different phases of reading learning. This seems to depend, in part, on the teaching method of the reading used. The objectives of this study were to identify and analyze the skills involved in the beginning of reading and writing in Early Childhood Education that predict the level of reading comprehension in Primary Education and know the differential weight that phonological awareness, naming speed and other skills involved in the reading process have during the first years of instruction depending on the method used. Therefore, a longitudinal study was carried out in which 130 students enrolled in educational centres located in the provinces of Castellón and Valencia. 52\% of them were studying according to the syntheticphonological method and $48 \%$ were following the analytic method. Instruments applied was RAN (Rapid Automatized Naming Test) and BIL (Initial Reading Program), and ACL (Reading Comprehension Test). The structural equation model has shown that in both methods the naming speed at 4 years has a significant effect on the variables related to the beginning of the reading at 5 years and these variables influence the reading comprehension of these children at 6 years. This indicates that naming speed constitutes the foundation of the phonological coding process on which the reader process is based.
\end{abstract}

Keywords: phonological awareness; alphabetic knowledge; naming speed; learning to read; RAN

\title{
INTRODUCCIÓN
}

La habilidad lectora es un proceso complejo integrado por distintas funciones cognitivas mediadas por diversas estructuras cerebrales, cuyo objetivo es el acceso al significado (Cuetos, 2009). La automatización de los procesos involucrados en el reconocimiento de palabras facilita la fluidez y precisión lectora, y libera los recursos atencionales para asignarlos a la comprensión. Es decir, a la elaboración de una representación mental significativa y coherente del texto.

A lo largo de los últimos 30 años se han identificado las habilidades y factores facilitadores que convierten a un niño en lector competente. Es una tarea necesaria para la atención temprana de los alumnos con dificultades en la adquisición del proceso lector. En este sentido, y atendiendo exclusivamente a los estudios de corte longitudinal, destacan como habilidades predictoras el conocimiento fonológico, el conocimiento alfabético y la velocidad de denominación (Sellés, 2008). Así mismo, dentro de la categoría de habilidades facilitadoras estarían el conocimiento metalingüístico acerca del lenguaje escrito y ciertas habilidades lingüísticas cuya base es el desarrollo del lenguaje oral. Esta categoría también integraría procesos cognitivos básicos como la atención, percepción y memoria (Eurydice, 2012). Todas ellas se adquieren y desarrollan a lo largo de la escolaridad. Sin embargo, no tienen el mismo grado de implicación en las distintas fases del aprendizaje lector 
(Rodríguez, van den Boer, Jiménez \& de Jong, 2015). Además, parecen estar moduladas por la transparencia de la lengua en la que se aprende a leer y del método de enseñanza de la lectura utilizado (Diuk \& Ferroni, 2013; Johnston \& Watson, 2005), ya que mientras que los métodos sintéticos fomentan el uso de estrategias de decodificación fonológica, los métodos analíticos potencian el desarrollo de estrategias de decodificación ortográfica. Así mismo, en lenguas transparentes la conciencia fonológica (CF) ha demostrado una mayor capacidad de predicción en los estadios iniciales del aprendizaje de la lectoescritura, cuando la adquisición del código aún no es completa. Sin embargo, conforme se consolida el dominio del código esa influencia parece tender a desaparecer (Onochie-Quintanilla et al., 2011; Defior \& Serrano 2011). Por otro lado, los estudios también han señalado una asociación entre la habilidad de denominación rápida y el rendimiento lector desde el momento en el que comienza el proceso de adquisición (Araújo, Reis, Petersson \& Faísca, 2015). La velocidad de nombramiento (VN) ha resultado ser un predictor consistente de la fluidez lectora en todas las ortografías y su capacidad predictiva se fundamenta en que ambas (VN y lectura) se nutren de procesos cognitivos compartidos (Landerl et al, 2019). En este sentido la VN parece estar vinculado al dominio lector de palabras reales, pseudopalabras y comprensión de textos a lo largo de toda la escuela primaria. Sin embargo, aún se desconoce la naturaleza exacta del proceso subyacente de dicha relación (Jones, Snowling \& Moll, 2016) y la implicación de esta a lo largo de las distintas etapas del proceso lector. Estas cuestiones han despertado el interés por conocer el grado de implicación de las funciones ejecutivas responsables de la lectura en las distintas fases del aprendizaje lector.

Así pues, los objetivos que persigue este estudio son dos: analizar e identificar qué habilidades implicadas en la lectura presentes al inicio de la lectoescritura que permiten predecir el nivel de comprensión lectora al inicio de la Educación Primaria. Y por otro lado, conocer el peso diferencial que la conciencia fonológica, la velocidad de nombramiento y otras habilidades implicadas en el proceso lector tienen en los dos primeros años del inicio de la lectoescritura, y si existen diferencias según el método empleado.

\section{MÉTODO}

\section{Participantes}

En el estudio participaron 130 alumnos (58.50\% chicas y $41.50 \%$ chicos) de tres colegios de la Comunidad Valenciana, de los que se obtuvieron medidas repetidas a los 4, 5 y 6 años, es decir cuando estaban cursando respectivamente $2^{\circ}$ y $3^{\circ}$ de Educación Infantil y $1^{\circ}$ de Educación Primaria. 63 de los alumnos participantes en el estudio pertenecían a un colegio cuyo método de enseñanza de la lectura era de tipo global, basado en la metodología de Glenn Doman (Doman, 2008) y otros 67 fueron iniciados en el aprendizaje de la lectura con un método fonológico-sintético (Letrilandia)(Usero, 2011). Todos los centros participantes eran de tipo privado-concertado con un nivel socioeconómico similar.

\section{Instrumentos}

Los instrumentos aplicados a los alumnos de 4 y 5 años fueron:

The Rapid Automatized Naming Test (RAN; Wolf \& Denkla, 2003) de aplicación individual. La tarea consiste en nombrar 200 estímulos agrupados en cuatro subtest (números, letras, colores e imágenes familiares), lo más rápidamente posible. Los estímulos de cada subtest se presentan en una cartulina diferente de $21 \times 30 \mathrm{~cm}$ organizados en 5 filas de 10 estímulos cada una. En cada subtest los estímulos son presentados aleatoriamente pero siempre 50 de cada uno de ellos. En el registro de la RAN se anota el tiempo que tarda el alumno en nombrar todos los estímulos de cada car- 
tulina y el número de errores que comete al nombrarlos. Con estos datos se obtiene un índice de eficiencia de cada subtest según el procedimiento descrito por Compton (2003) que consiste en convertir las puntuaciones en números/segundo, letras/segundo, colores/segundo e imágenes/segundo. La fiabilidad medida con el alfa de Cronbach es de 0,809.

La Batería de Inicio a la Lectura para niños de 3 a 6 años BIL (Sellés, Martínez, Vidal-Abarca \& Gilabert, 2008) incluye 15 pruebas organizadas en 5 escalas que miden la conciencia fonológica (mediante las subescalas "rimas", "contar palabras", "contar sílabas", "aislar sílabas y fonemas" y "omitir sílabas"), el conocimiento alfabético (a través del subtest de "conocimiento del nombre de las letras"), el conocimiento metalingüístico (mediante las subescalas "reconocer palabras", "reconocer frases", "funciones de la lectura"), las habilidades lingüísticas (a través de los subtest "vocabulario", "articulación", "conceptos básicos" y "estructuras gramaticales") y procesos cognitivos (mediante las subescalas "memoria secuencial auditiva" y "percepción visual". En cuanto a la fiabilidad de la prueba, el Alpha de Cronbach de todas pruebas oscila entre 0.55 y 0.97.

A los alumnos de 6 años se les aplicó:

El test de Comprensión Lectora para niños de 1a a 6ํํㄹ de Educación Primaria ACL (Catalá, Catalá, Molina \& Monclús, 2001). En este estudio se ha utilizado el primer nivel (ACL-1). El instrumento ofrece información de cuatro escalas: la comprensión literal, reorganizativa, inferencial y crítica. La fiabilidad medida con el coeficiente KR-20 es de 0.80 para ACL-1.

\section{Procedimiento}

Tras obtener los permisos oportunos se pidió a cada colegio que enviara a los padres una carta en la que se explicaba brevemente la investigación y se les solicitaba el consentimiento para que participaran sus hijos. Los instrumentos de evaluación (RAN, BIL y ACL) fueron administrados de forma individual en diferentes sesiones de 30 minutos de duración por cada alumno. La respuesta de los alumnos fue de colaboración e interés. Todos los evaluadores recibieron formación específica para asegurar que ofrecieran las mismas directrices en la administración de los instrumentos de evaluación.

\section{RESULTADOS}

Se realizaron unos Modelos de Ecuación Estructural Multigrupo con el fin de analizar simultáneamente la potencia predictora de las variables medidas y comparar su fuerza en los dos métodos de enseñanza de la lectura utilizados en los colegios participantes del estudio. Se probaron cuatro modelos y se eligió el que mejor ajustó atendiendo a índices como ${ }^{2}$ de Satorra-Bentle), IFI (índice de ajuste incremental), TLI (índice de Tucker-Lewis) y RMSEA (raíz del residuo cuadrático promedio de aproximación). Atendiendo a las relaciones significativas halladas entre las variables evaluadas se desarrolló el modelo que se muestra a continuación, según el cual, la Velocidad de Nombramiento medida a los 4 años (RAN1) tenían un efecto significativo en las variables relacionadas con el inicio de la lectura evaluadas a los 5 años (BIL2), las cuales, a su vez, influirían en la comprensión lectora presentada por los niños a los 6 años de edad (ACL).

A continuación, se presenta el modelo resultante en el método global (Figura 1) y en el método fonológico-sintético (Figura 2), y la tabla con los valores de bondad de ajuste de este modelo (Tabla 1). 
Figura 1. Representación gráfica del modelo en el método global.

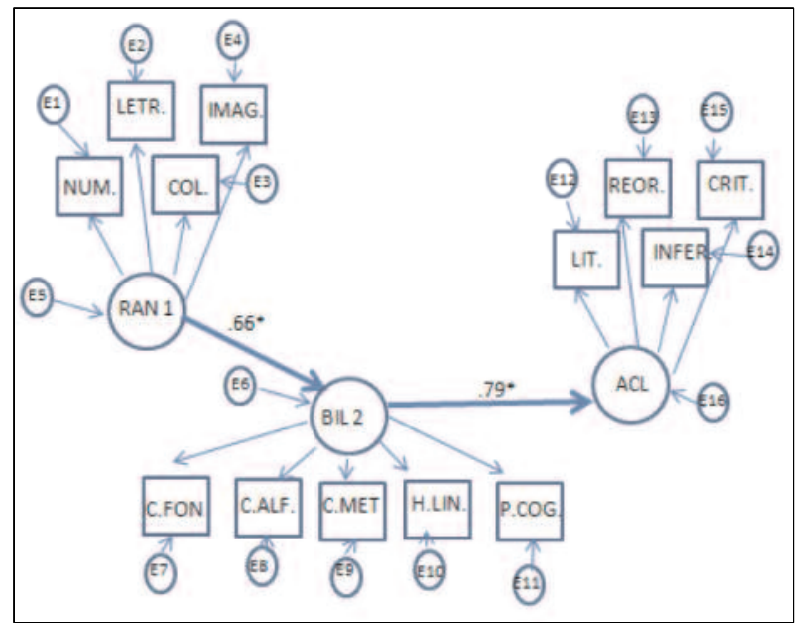

Figura 2. Representación gráfica del modelo en el método sintético.

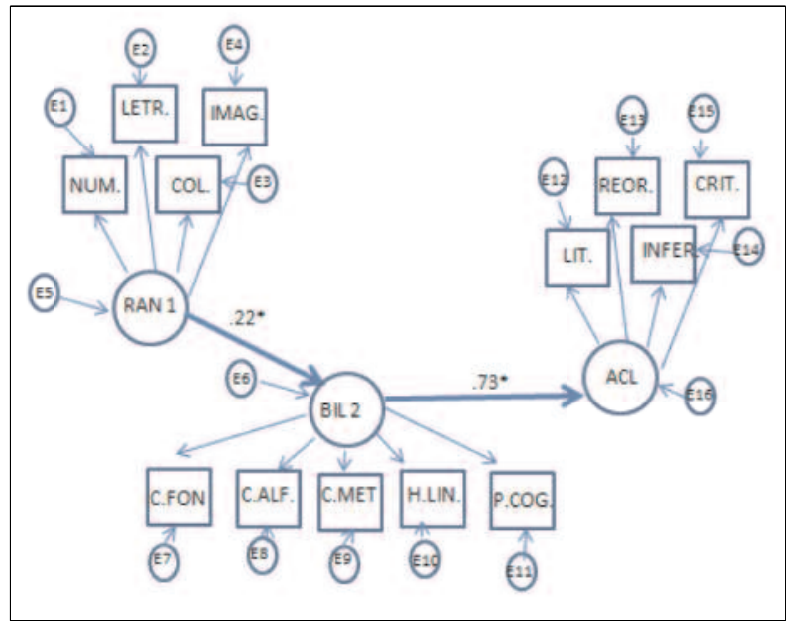

Tabla 1

Índices de bondad de ajuste del modelo

\begin{tabular}{cccccccc}
\hline $\boldsymbol{\chi}^{\mathbf{2}}$ & g.l. & $\mathbf{P}$ & $\boldsymbol{\chi}^{\mathbf{2} / \text { g.l. }}$ & NNFI/TLI & RMSEA & CFI & IFI \\
\hline 206.64 & 126 & $<0.001$ & 1.63 & 0.87 & 0.07 & 0.89 & 0.90 \\
\hline
\end{tabular}

Nota: $\chi^{2}=\chi^{2}$ de Satorra-Bentler; $\chi^{2} /$ G.L. $=\chi^{2}$ relativo; NNFI/TLI= Estadístico de bondad de ajuste relativo; RMSEA= Raíz cuadrada media del error de aproximación; CFI= Índice de ajuste comparativo; IFI= Índice de ajuste incremental. 
Como se puede comprobar en las figuras 1 y 2, este modelo confirma los supuestos desarrollados a la hora de ponerlo a prueba. Efectivamente, tanto en el método global, como en el fonológico-sintético la RAN en su primera medida (a los 4 años) se relaciona de forma estadísticamente significativa con las variables de la BIL evaluadas a los 5 años, las cuales predicen significativamente la comprensión lectora a los 6 años.

Además, como se puede observar en la tabla 1, el ajuste presentado por éste modelo se puede considerar satisfactorio ( $\mathrm{TLI}=.87 ; \mathrm{CFI}=.89 ; \mathrm{IFI}=.90 ; \mathrm{RMSEA}=.07$ ). Por todo ello se considera que este modelo es el que mejor explica los datos, las variables de estudio y sobre todo las relaciones entre ellas. Por ello se analizan con más detalle los elementos que componen el modelo y las posibles diferencias existentes entre los dos métodos de enseñanza de la lectura.

Tal como se recoge en la tabla 2, todas las escalas analizadas se relacionan de forma estadísticamente significativa con las variables que las componen (RAN con RAN números (NUM), RAN letras (LETR), RAN colores (COL) y RAN imágenes (IMAG); BIL con conciencia fonológica (C.FON), conocimiento alfabético (C.ALF), conocimiento metalingüístico (C.MET), habilidades lingüísticas (H.LING) y procesos cognitivos (P.COG) y ACL con comprensión literal (LIT), reorganizativa (REOR), inferencial (INFER) y crítica (CRIT), como es esperable. Además, como se ha mencionado, la Velocidad de nombramiento medida a los 4 años (RAN 1) se relaciona con la madurez lectora en 5 años (BIL2) $(p=.003 ; p<.001)$ que, a su vez, predice la comprensión lectora a los 6 años (ACL) $(p=.002 ; p<.001)$.

Tabla 2

Relación entre variables del modelo y diferencias entre métodos de enseñanza de la lectura

\begin{tabular}{|c|c|c|c|c|c|}
\hline \multirow[b]{2}{*}{$\begin{array}{l}\text { Variables } \\
\text { relacionadas }\end{array}$} & \multicolumn{2}{|c|}{ Método Global } & \multicolumn{2}{|c|}{ Método fonológico-sintético } & \multirow[t]{2}{*}{ z-score } \\
\hline & Estimación & $\mathbf{p}$ & Estimación & $\mathbf{p}$ & \\
\hline RAN1 $\rightarrow$ BIL2 & .66 & .003 & .22 & $<.001$ & $1.584 *$ \\
\hline BIL2 $\rightarrow$ ACL & .79 & .002 & .73 & $<.001$ & 1.330 \\
\hline RAN1 $\rightarrow$ NUM & .79 & $<.001$ & .89 & $<.001$ & 1.365 \\
\hline RAN1 $\rightarrow$ LETR & .92 & $<.001$ & .93 & $<.001$ & 1.398 \\
\hline $\mathrm{RAN1} \rightarrow \mathrm{COL}$ & .60 & $<.001$ & .88 & $<.001$ & 1.863 \\
\hline RAN1 $\rightarrow$ IMAG & .60 & $<.001$ & .84 & $<.001$ & 1.333 \\
\hline BIL2 $\rightarrow$ C.FON & .48 & .002 & .67 & .002 & 1.264 \\
\hline BIL2 $\rightarrow$ C.ALF & .70 & .002 & .45 & .002 & $2.244 * *$ \\
\hline BIL2 $\rightarrow$ C.MET & .69 & .001 & .55 & $<.001$ & 1.542 \\
\hline BIL2 $\rightarrow$ H.LIN & .56 & .002 & .81 & $<.001$ & .798 \\
\hline BIL2 $\rightarrow$ P.COG & .44 & .008 & .65 & $<.001$ & .741 \\
\hline ACL $\rightarrow$ LIT & .89 & $<.001$ & .80 & $<.001$ & .986 \\
\hline ACL $\rightarrow$ REOR & .66 & $<.001$ & .63 & $<.001$ & 1.032 \\
\hline ACL $\rightarrow$ INFER & .87 & $<.001$ & .72 & $<.001$ & .178 \\
\hline $\mathrm{ACL} \rightarrow \mathrm{CRIT}$ & .72 & $<.001$ & .68 & $<.001$ & .041 \\
\hline
\end{tabular}

Nota: $* \mathrm{p}<.05 ; * * \mathrm{p}<.001$

En cuanto a las diferencias entre los dos métodos de enseñanza de la lectura estudiados, podemos señalar que el z-score resulta ser estadísticamente significativo en la relación entre la velocidad de nombramiento en 4 años (RAN 1) y la madurez lectora en 5 años (BIL 2) (1.584*), y entre la madurez lectora en 5 años (BIL 2) y el conocimiento alfabético (C.ALF $=\left(2.244^{* *}\right)$, en ambos casos a favor del método fonológico-sintético. Esto podría apuntar a una probable relación de cau- 
salidad en dicho método, entre la velocidad de nombramiento medida a los 4 años y la madurez lectora evaluada a los 5 años con la prueba BIL.

Así pues, los resultados permiten destacar un efecto de la velocidad presente en los niños de 4 años en su nivel de aprendizaje de la lectura en 5 años. Este último, además, resulta ser predictivo de la comprensión lectora que esos mismos niños presentan a los 6 años de edad. En este sentido parece que la influencia de la velocidad de nombramiento sobre la comprensión podría estar mediada por el conjunto de variables vinculadas con el inicio de la lectura, por lo que no sería una relación directa y lineal.

Finalmente, analizando la diferencia entre el método global y el fonológico-sintético, no se han encontrado diferencias significativas en la capacidad de predicción de las variables medidas con la BIL en ambos métodos, ya que ambos predicen la comprensión lectora. Sin embargo, sí se han hallado diferencias entre el peso de la relación entre la velocidad de nombramiento en 4 años (RAN1) y la madurez lectora en 5 años (BIL2) y entre la madurez lectora en 5 años (BIL2) y conocimiento alfabético, demostrando que dichas relaciones tienen un peso mayor en el método fonológico-sintético. Estos resultados confirman que variables relacionadas con el conocimiento de los sonidos como el conocimiento alfabético tienen más importancia en el método fonológico-sintético.

\section{DISCUSIÓN}

Los resultados obtenidos en cuanto a las habilidades que mejor predicen la comprensión lectora en el primer curso de Educación Primaria muestran la asociación que existe entre VN y CF y CA, que a su vez son predictoras de la comprensión lectora. Según los datos que ofrece el modelo de ecuación estructural, parece que el nivel de desarrollo que poseen los niños a los 4 años en las variables CF y CA no es tan determinante en el grado de comprensión lectora que presentarán dos años más tarde como la velocidad de nombramiento, la cual se ha revelado como una variable de peso que influye en la CF y el CA, predictoras ambas de la comprensión lectora. Existen múltiples evidencias no sólo acerca de la importancia de la VN en el desarrollo de la habilidad lectora (Kirby, Roth, Desrochers y Lai, 2008; Onochie-Quintanilla et al., 2011), sino de la asociación que existe entre la VN y el rendimiento lector desde el momento en el que comienza el proceso de adquisición (Araújo et al., 2015).

En este sentido, parece razonable que la VN mantenga una estrecha relación con las variables que a los 5 años sí han demostrado ser predictoras de la comprensión lectora independientemente de si la medida de alfabetización se basa más en las habilidades de codificación fonológica (potenciadas por el método sintético) u ortográfica (potenciadas por el método global) (Georgiou, Parrila, Kirby \& Stephenson, 2008; Guardia, 2003; López-Escribano y Katzir, 2008). Además indica que en el desarrollo evolutivo del niño la VN parece ser una variable de más temprana adquisición que la CF o el CA debido en parte a su carácter de variable de procesamiento fonológico implícito (Defior \& Serrano, 2011), lo que comportaría un desarrollo anterior al de las variables explícitas, ya que su adquisición exige menos manipulación consciente por parte del niño, y por lo tanto menos conocimientos. Todo ello indicaría la importancia de consolidar la velocidad de nombramiento previamente para que la conciencia fonológica pueda desarrollarse adecuadamente, además de la cuestión de que tanto una como otra son imprescindibles para alcanzar buenos niveles de comprensión lectora en cursos posteriores.

Estos resultados guardan relación con las aportaciones de Ziegler y Goswami (2005) quienes afirman que las habilidades fonológicas son una consecuencia de aprender a leer y, por tanto son posteriores en su adquisición. Así pues, la conciencia fonológica se desarrolla una vez que los niños han adquirido cierto nivel de desarrollo en el aprendizaje de la lectura y la escritura. Además, se con- 
firma de nuevo la cuestión de que componentes como la velocidad de nombramiento tienen más peso a edades inferiores mientras que las habilidades relacionadas más directamente con la lectura irían ganando peso con el avanzar de la edad.

En cuanto al objetivo de analizar el peso diferencial que la conciencia fonológica, la velocidad de nombramiento y otras habilidades implicadas en el proceso lector tienen durante los dos primeros años del inicio de la lectoescritura y si existen diferencias en esto según el método empleado. El modelo indicó que, independientemente del método de lectura utilizado en la enseñanza de la lectura, la VN a los 4 años predice la conciencia fonológica, el conocimiento del alfabeto, habilidades lingüísticas, metalingüísticas y los procesos cognitivos medidos a los 5 años, que a su vez predicen la comprensión lectora que adquieren dichos alumnos a los 6 años de edad. Es decir, independientemente de si la medida de alfabetización utilizada se basa más en las habilidades de codificación fonológica (potenciadas por el método sintético) u ortográfica (potenciadas por el método global) (Georgiou et al., 2008; Guardia, 2003; López-Escribano \& Katzir, 2008), no se han encontrado diferencias significativas en la capacidad de predicción de las variables que influyen en la comprensión lectora. Por el contrario, sí se han encontrado diferencias en el peso de las relaciones entre algunas variables dentro del método sintético. Concretamente, entre la velocidad de nombramiento (medida a los 4 años) y la madurez lectora (medida a los 5 años), y entre la madurez lectora (medida a los 5 años) y el conocimiento alfabético (medido a los 5 años), las cuales indican que el método sintético favorece la relación entre dichas variables, lo que demuestra que el método de instrucción sí modula el curso del desarrollo de las habilidades cognitivas que subyacen a la lectura.

En cuanto a las diferencias entre los modelos predictivos generados a partir de las variables existentes a los 4 y 5 años se ha encontrado que a los 4 años la variable con mayor capacidad predictiva del proceso lector sería la velocidad de nombramiento mientras que a los 5 años sería la conciencia fonológica. A la vista de los resultados obtenidos en nuestros análisis se confirma que tanto en el método sintético como en el global, a los 4 años, la velocidad de nombramiento ha resultado ser la única variable predictora de las variables determinantes del proceso lector a los 5 años, es decir de la conciencia fonológica, conocimiento alfabético, conocimiento metalingüístico, habilidades lingüísticas y los procesos cognitivos, señalando que dicha relación parece más fuerte en el método sintético. Así mismo la CF, medida a los 5 años, aunque no únicamente ella, ha resultado predictora de la comprensión lectora a los 6 años.

Por último, en cuanto a diferencias entre modelos los resultados obtenidos en nuestro estudio no se observa una capacidad predictiva diferente. Ambos métodos predicen de forma significativa la comprensión lectora. Tanto en el método sintético como en el global, la VN medida a los 4 años se relaciona con la madurez lectora a los 5 años, y ésta, a su vez, predice la comprensión lectora a los 6 años. En lo que sí difieren, como señalamos anteriormente, es en el peso de la relación entre algunas variables dentro del método, concretamente a favor del método sintético. Sin embargo, este dato no afecta a la capacidad predictiva de ambos métodos, quienes han resultado predictores de la comprensión lectora en igual medida. Esto indica que el método de instrucción utilizado en la enseñanza de la lectura no determina la capacidad predictiva de las variables sino más bien la edad a la que resulta prioritario haber desarrollado determinada habilidad, ya sea a través de aproximaciones sintéticas 0 analíticas.

\section{REFERENCIAS BIBLIOGRÁFICAS}

Araújo, S., Reis, A., Petersson, K. M., \& Faísca, L. (2015). Rapid automatized naming and reading performance: A meta-analysis. Journal of Educational Psychology, 107(3), 868.

Català, G., Català, M.; Molina, E. \& Monclús, R. (2001). Evaluación de la Comprensión Lectora. 
Pruebas ACL (1-6 grado de primaria). España: Editorial Graó.

Compton, D. L. (2003). Modeling the relationship between growth in rapid naming speed and decoding skill in first-grade children. Journal of Educational Psychology, 95, 225-239.Cuetos, F. (2010). Psicología de la lectura. Madrid: Wolters Kluwer España.

Cuetos, F. (2010). Psicología de la lectura. Madrid: Wolters Kluwer España. Defior, S. \& Serrano, F. (2011). Procesos fonológicos explícitos e implícitos. Lectura y Dislexia. Revista de Neuropsicología, Neuropsiquiatría y Neurociencias, 11, 79-94.

Doman, G. (2008). Cómo enseñar a leer a su bebé. Madrid: EDAF.

Diuk, B. \& Ferroni, M. (2013). ¿Anglocentrismo en los modelos de adquisición lectora? Un estudio en una lengua de ortografía transparente. Summa psicológica UST, 10(2), 29-39.

Eurydice. Ministerio de Educación, Cultura y Deporte (2012). La atención al alumnado con dislexia en el sistema educativo en el contexto de las necesidades específicas de apoyo educativo. España: Colección Eurydice España-Redie. Recuperado 6 febrero, 2016 de https://sede.educacion.gob.es/publiventa/la-atencion-al-alumnado-con-dislexia-en-el-sistema-educativo-en-elcontexto-de-las-necesidades-especificas-de-apoyo-educativo/educacion-especial-y-compensatoria/15956

Georgiou, G. K., Parrila, R., Kirby, J. R., \& Stephenson, K. (2008). Rapid naming components and their relationship with phonological awareness, orthographic knowledge, speed of processing, and different reading outcomes. Scientific Studies of Reading, 12, 325-350.

Guardia, P. (2003). Relaciones entre habilidades de alfabetización emergente y la lectura, desde el nivel transición mayor a primero básico. Psykhe,12(2), 63-79.

Jones, M., Snowling, M. \& Moll, K. (2016). What automaticity deficit? Activation of lexical information by readers with dyslexia in a RAN-Stroop task. Journal of Experimental Psychology: Learning, Memory, and Cognition, 42, 465-474.

Johnston, R. S., \& Watson, J. E. (2005). A seven year study of the effects of synthetic phonics teaching on reading and spelling attainment. Edinburgh, UK: Scottish Executive Education Department, Information, Analysis and Communication Division.

Kirby, J. R., Roth, L., Desrochers, A., \& Lai, S. (2008). Longitudinal predictors of word reading development. Canadian Psychology, 49, 103-110.

Landerl, K., Freudenthaler, H.H., Heene, M., De Jong, P.F., Desrochers, A., Manolitsis, G., Parrila, R. \& Georgiou, G.K. (2019). Phonological Awareness and Rapid Automatized Naming as Longitudinal Predictors of Reading in Five Alphabetic Orthographies with Varying Degrees of Consistency, Scientific Studies of Reading, 23 (3), 220-234.

López-Escribano, C. \& Katzir, T. (2008). Are phonological processes separate from the processes underlying naming speed in a shallow orthography? Education Psychology, 16, 641-666.

Onochie-Quintanilla, E., Simpson, I, Caravolas, M. \& Defior, S. (2011). Letter knowledge, phoneme awareness and RAN as predictors of reading fluency in Spanish. 10th Symposium of Psycholinguistics, San Sebastián, April 13th-16th.

Rodríguez, C., Van den Boer, M., Jiménez, J.E. \& de Jong, P.F. (2015) Developmental Changes in the Relations Between RAN, Phonological Awareness, and Reading in Spanish Children. Scientific Studies of Reading, 19 (4), 273-288.

Sellés, P. (2008). Elaboración de una prueba de habilidades relacionadas con el desarrollo inicial de la lectura (BIL 3-6). Tesis. Universitat de Valencia, Valencia.

Sellés, P., Martínez, T., Vidal-Abarca, E., \& Gilabert, R. (2008). Batería de inicio a la lectura para niños de 3 a 6 años. Madrid: ICCE.

Usero Alijarde, A. (2011). Letrilandia. Libros de lectura. Propuesta didáctica. Zaragoza: Edelvives. 
Wolf, M., \& Denckla, M. (2003). Rapid Automatized Naming Tests. Greenville, SC: Super Duper.

Ziegler, J. \& Goswami, U. (2005). Reading acquisition, developmental dyslexia, and skilled reading across languages: A psycholinguistic grain size theory. Psychological Bulletin, 131, 3-29. 